\title{
The metabolic effects of exercise
}

\section{By Petrr Harris, Department of Medicine, University of Birmingham, Queen Elizabeth Hospital, Birmingham}

The metabolic effects of exercise have classically been considered in terms of the changing composition of the blood and it may be thought a little old-fashioned to perpetuate this approach. Nevertheless, I believe that the changes in the composition of the blood during exercise are more complex than has generally been credited and the time is opportune to re-examine them.

The increase in the concentration of lactate is historically the first observation and the one which has excited the most interest. The classical theory of oxygen debt introduced by A. V. Hill held the expenditure of energy by the exercising muscle to exceed the energy available to it from oxidative catabolism, the balance or debt being made up by the anaerobic production of lactate. After exercise, the oxygen consumption remained high for a period of time and this was thought to be due to the combustion of the lactate which resulted in the repayment of the debt. Later observations made it necessary to modify the simplicity of the original concept. Early on, Margaria, Edwards \& Dill (r933), for instance, divided the oxygen debt into a lactic and an alactic phase, and the most recent modification has been the calculation of 'excess lactate' by Huckabee (1958a,b).

Such modifications are necessitated by discrepancies between fact and theory; but fact must accompany theory, and, although it may seem surprising, through nearly half a century of investigation the simple observation of how the concentration of lactate in the blood changes during the course of exercise has only been made by a handful of authors. Such few observations as have been published record conflicting results (Bang, 1936; Lundin \& Ström, 1947; Asmussen, 1950) and it is for this reason we have made a number of studies on the concentration of lactate in arterial blood during exercise.

During the course of $30 \mathrm{~min}$ mild bicycling exercise in the recumbant position, it is usual for the concentration of lactate to rise rapidly for the first few minutes, reaching a maximum after about $5^{-10} \mathrm{~min}$. From this time onwards it usually falls progressively and this decline is often not affected by the moment of stopping exercise. Such changes in the concentration of lactate occur while the external work, the ventilation and the oxygen uptake remain constant.

If the subject bicycles for only $4 \mathrm{~min}$, the concentration of lactate in the arterial blood follows a very similar course, reaching a maximum about 5-10 min after the beginning of exercise and then declining gradually. In this instance the concentration of lactate continues to rise after the end of exercise and remains high long after the oxygen uptake has returned to its normal resting level.

Such simple observations raise doubts about whether there is any relation between the increased uptake of oxygen after exercise and the concentration of lactate in the blood. Nor are such doubts dispelled by considering the lactate:pyruvate ratio or the 'excess lactate', both of which quantities can be shown to move independently of the oxygen uptake under such circumstances. 
And yet the concentration of lactate $5^{-10} \mathrm{~min}$ after the beginning of a $10 \mathrm{~min}$ period of exercise can be shown to correlate with the oxygen uptake during exercise, and so can the concentration of pyruvate and the lactate:pyruvate ratio (Harris, Bateman \& Gloster, 1962a). Whether, on the other hand, such correlations imply a causative link is open to doubt, and it may simply be that the oxygen uptake, the production of lactate and pyruvate and their ratio are all independently related to the magnitude of the exercise.

Several authors have made calculations relating the concentration of lactate in the blood to the uptake of oxygen, but implicit in all such calculations is the assumption that lactate is produced during exercise and only metabolized subsequently. Studies of regional arterio-venous differences in the concentration of lactate during exercise have shown how untenable such a view is (Harris, Bateman \& Gloster, I $962 b$ ). For, although lactate is being produced by the legs during bicycling exercise, it is simultaneously being metabolized by the liver, by the heart and by those muscles which are not partaking in the physical activity.

All these observations tend to break those links which teaching and long habit of thought have formed in our minds between the oxygen uptake and the concentration of lactate in the blood. And, if one is prepared to doubt the payment of an oxygen debt by lactate, it is only one step further to doubt whether the concept of an oxygen debt is itself meaningful.

It would indeed be surprising if a 'square-wave input', such as is constituted by a period of exercise, would produce an equally square-wave response in so complex a physical and chemical system as the body, and the increased uptake of oxygen after exercise may be understandable simply in such terms.

Although lactate and pyruvate have been the metabolites which have most interested those studying exercise, the concentration of other substances in the blood may also be considerably affected. The concentration of glucose, for instance, may fall slightly at the beginning of exercise but shows a characteristic increase after exercise has finished. This is one of a number of metabolic events specifically associated with the moment of stopping exercise. Studies of the arterio-venous difference in the concentration of glucose in various regions of the body suggest that, during exercise, there is a decreased utilization of glucose by inactive skeletal muscle and by the brain. It appears even possible that inactive muscle can liberate glucose into the blood stream under these conditions (Harris, Bateman \& Gloster, I962c).

The concentration of free fatty acids in the blood is also affected by exercise, tending to fall somewhat at the beginning of physical activity and to increase substantially after the moment of stopping (Harris, Fletcher, Gloster \& Gotsman, 1965). The studies of Havel, Naimark \& Borchgrevink (1963) have shown that exercise is accompanied both by an increased rate of lipolysis and an increased rate of utilization of free fatty acids by the active muscles. The rise in concentration of free fatty acids after the end of exercise occurs when the rate of utilization suddenly falls, unmasking the increased rate of lipolysis.

When triglycerides are broken down in adipose tissue to form free fatty acids, glycerol is also liberated. The concentration in the blood does not change greatly, 
however, until the end of exercise, when it rises in a fashion similar to the free fatty acids (Harris et al. 1965). Presumably the reasons for this are similar to those which underlie the changes in the free fatty acids.

Such simple observations serve to illustrate how very complex is the metabolic response of the body to physical activity. At a more practical level they also show how important is the timing of sampling in any study designed to relate the concentration of a metabolite in the blood with some other physiological measurement during exercise.

\section{REFERENCES}

Asmussen, E. (1950). Acta physiol. scand. 20, 125.

Bang, O. (1936). Skand. Arch. Physiol. 74, Suppl. 1o, p. 49.

Havel, R. J., Naimark, A. \& Borchgrevink, C. F. (1963). F. clin. Invest. 42, 1054.

Harris, P., Bateman, M. \& Gloster, J. (r962a). Clin. Sci. 23, 53 I.

Harris, P., Bateman, M. \& Gloster, J. (r962b). Clin. Sci. 23, 545.

Harris, P., Bateman, M. \& Gloster, J. (1962c). Clin. Sci. 23, 561 .

Harris, P., Fletcher, R. F., Gloster, J. \& Gotsman, M. (1965). Clin. Sci. 28, 343.

Huckabee, W. E. (1958a). \%. clin. Invest. 37, 244.

Huckabee, W. E. (1958b). \%. clin. Invest. 37, 255.

Lundin, G. \& Ström, G. (1947). Acta physiol. scand. 13, 253.

Margaria, R., Edwards, H. T. \& Dill, D. B. (1933). Am. F. Physiol. ro6, 689.

\section{Nutrition and its relation to body composition in exercise}

\section{By Jana Paर̌ízková, Physical Culture Research Institute, Prague, Czechoslovakia}

Increased energy output due to enhanced physical activity in sportsmen requires correspondingly increased calorie input which reaches $7000-8000 \mathrm{kcal}$ per day in special kinds of sports disciplines during most intensive periods of training. Such an increased input does not result, as it surely would in subjects of an average population, in enhanced body fatness; on the contrary, typical body composition, i.e. a high ratio of lean body mass at the expense of fat, is one of the most remarkable features of the physique of top athletes. Matiegka (I92I) was the first who evaluated these characteristic differences by means of anthropometric measurements. By a special method, densitometry, the body composition of sportsmen was studied by Behnke, Feen \& Welham (1942) and Welham \& Behnke (1942). In their work it was shown that even in instances of increased relative body-weight which in subjects not systematically interested in sports characterizes enhanced development of fat, the body of an athlete contains only a very low ratio of adipose tissue and the increased weight is due to highly developed muscle mass.

Not only sport but also different professional activity could cause differences in the ratio of body fat to lean body mass, which was demonstrated in subjects from various countries by Brožek (I956) and Keys \& Brožek (1957). Tanner (1952) elucidated the differences of physique in trained and untrained subjects by anthropometric methods. In men during military service trained to different work loads the lowest skinfold thicknesses were always found in men exposed to greatest physical strain (Koldovský, Pařízková, Špaček \& Hahn, I959). In the Soviet Union the 